\title{
Retrait des terres et subsidiarité dans le cadre de la Politique Agricole Commune
}

\author{
Pierre-Alain Jayet \\ INRA - Unité d'Economie - Centre de Versailles-Grignon
}

Gilles Rotillon

THEMA - Université de Paris X - Nanterre

\section{$1 \quad$ Introduction}

L'incitation ou l'obligation du retrait des terres de la production agricole est l'un des modes de régulation des marchés agricoles adopté depuis plusieurs décennies aux États-Unis et plus récemment en Europe. Elle concerne aussi bien des productions annuelles telles que les céréales; que des productions pérennes comme la vigne, même si les modalités précises diffèrent quelque peu selon la géographie et les productions concernées. Ainsi, une politique de gel peut elle s'accompagner d'une interdiction de produire sur les surfaces en cause, ou bien cette politique peut ne constituer qu'un "arrachage" temporaire suivi d'un réencépagement -- pour la production de vin de qualité notamment.

A titre d'exemple, en matière viticole, l'Union Européenne (UE) a offert des primes variant de 1449 à 12317 ECU/ha dès lors que le producteur bénéficiaire s'engageait à retirer de la production au moins $20 \%$ de ses terres. Entre les campagnes 1988/89 et 1996/97, ce sont 98100 ha en France et 490000 ha dans l'UE à 15 qui ont été «gelés », sur des totaux respectifs de surfaces plantées en vigne de 912000 ha et 3428000 ha (moyenne sur les années 1993-1997). Une modification de l'organisation commune de marché (OCM) est intervenue à partir de la campagne 1996/97. L'arrachage de la vigne a néanmoins représenté un coût de $232 \mathrm{M}$ ECU supporté par le budget de l'UE en 1997, l'OCM viti-vinicole représentant au total un coût européen de $970 \mathrm{M}$ ECU.

Dans le secteur dit des grandes cultures (céréales et oléagineux principalement), le gel de terre a fait partic des instruments de l'intervention 
plus tardivement en Europe (en 1988) qu'aux États Unis (dans les années 30). La question de laisser les États disposer d'une marge dans les modalités de l'intervention (en matière de prime à l'hectare en particulier) s'est rapidement posée. Le problème de l'application du principe de subsidiarité est alors posé.

Par le FAIR Act aux États-Unis (Federal Agricultural Improvement and Reform,1996) ou avec l'Agenda 2000 qui redéfinit la politique agricole de l'Union Européenne, et dans l'esprit des accords de l'Organisation mondiale du commerce, les politiques de soutien des prix et plus généralement les politiques de soutien à la production tendent à être limitées, ainsi que le gel de terres qui en était le corollaire. Néanmoins, en Europe en particulier, cette option n'est pas abandonnée.

L'application effective d'une politique européenne de retrait des terres renvoit à l'hétérogénéité des pays dans lesquels elle serait appliquée. Le principe du marché agricole unique qui régit les OCM n'est pas incompatible avec une politique de régulation faisant jouer la subsidiarité. Historiquement, par exemple lors de la politique de gel instaurée en 1988, la prime proposée pouvait varier dans une plage de valeurs autorisées à l'échelle européenne, à condition que le coût soit en partie supporté par l'Etat-membre si la prime nationale dépasse la prime européenne.

Dans cet article, nous nous proposons de comparer à l'échelle de l'UE les avantages respectifs d'une politique de subsidiarité et d'une politique uniforme s'appuyant sur le gel de terres. Nous utiliserons un critère d'efficacité social fondé sur la somme pondérée des variations de surplus affectant les producteurs, les consommateurs et les contribuables. Nous nous limiterons à l'une des options de gel contractuel abordées par ailleurs (Jayet, 2001), sous la forme d'un contrat simple, dans un cadre d'information asymétrique entre le régulateur et les producteurs. Sur ce dernier point, nous nous appuyons sur la théorie des incitations et nous suivons la méthode d'analyse initiée par Baron et Myerson (1982). Rappelons que la pertinence d'une politique de gel de terre associée à une politique de prix garanti a été démontrée en plusieurs occasions dans un cadre de second rang (Gisser, 1993, Bourgeon et al., 1995), ce que ne remet pas en cause le modèle plus simple de représentation des systèmes de production que nous reprenons ici.

Le problème est étudié OCM par OCM. La réforme de la politique agricole est présentée comme un jeu en trois étapes où interviennent les autorités de régulation communautaire et nationale, et les producteurs agricoles. En première étape, la Commission Européenne décide du niveau du prix garanti et de l'application ou non du principe de subsidiarité. En seconde étape, les autorités en charge de la définition des contrats proposent un contrat de gel de terre simultanément à tous les producteurs dont ils ont la charge. Dans le cas où le principe de subsidiarité est appliqué, on suppose que les États membres déterminent simultanément le contrat de gel de terre qu'ils proposent à leurs producteurs dans un jeu non coopératif à la Nash. En dernière étape, les producteurs ajustent leur niveau de production con- 
naissant le prix européen et le contrat national de gel qui leur sont proposés. Dans le cas d'une politique unique de gel de terres, la dernière étape est inchangée, tandis qu'en seconde étape l'UE choisit le contrat unique européen de gel de terre.

Le papier est organisé comme suit. La section 2 traite de la détermination des contrats optimaux dans la situation d'asymétrie d'information qui caractérise la question étudiée. Dans la section 3 nous nous intéressons à la dernière étape du jeu non-coopératif, en déterminant, à prix fixé, les contrats optimaux dans le cas de la subsidiarité en contrats. La section 4 est consacrée au cas du contrat unique européen et la section 5 traite de la comparaison des bien-être sociaux correspondant aux deux modalités contractuelles, le prix garanti étant supposé identique. On peut alors étudier la première étape du jeu dans la section 6 où sont calculés et comparés les prix optimaux. La section 7 propose une application empirique de ces résultats à la régulation du marché européen du blé tendre dans l'UE-12, et la section 8 conclut et indique des prolongements possibles de ce travail.

\section{Détermination des contrats}

La subsidiarité est définie par rapport au niveau de décentralisation du choix du contrat. Ou bien le contrat est décentralisé au niveau des Étatsmembres, ou bien il est choisi de manière uniforme à l'échelle de l'Union Européenne. Dans le premier cas, les transferts sont à la charge des budgets nationaux, dans le second les transferts sont à la charge du budget européen. Quelle que soit l'échelle, la sélection du contrat repose sur une même forme de mécanisme incitatif, détaillée dans cette section. On identifiera l'échelle et l'Etat-membre par un indice $k \in \mathcal{K}=\{1,2, \ldots, K\}$. Le contrat unique européen sera indexé par « $U E$ ». Le mécanisme à la base du contrat de gel est alors identique au mécanisme décrit par Jayet (2001), et l'on en rappelle les fondements.

On considère des exploitations agricoles, en nombre $S_{k}$, produisant un même bien et de surface égale à l'unité (pour les pays non producteurs, $S_{k}=0$ ). Chaque exploitation est caractérisée par son rendement $\theta$, qui est par définition le rendement moyen de l'exploitation. Les rendements sont distribués selon une densité $f_{k}(\theta)$ et une répartition $F_{k}(\theta)$ définies sur $\left[0, \infty\left[\right.\right.$. On supposera la densité positive sur un support $\left[\underline{\theta}_{k}, \bar{\theta}_{k}\right]$ lorsque le pays $k$ est producteur (avec $0<\underline{\theta}_{k}<\bar{\theta}_{k}<\infty$ ), et nulle en dehors de cet intervalle ou si le pays est uniquement consommateur. On supposera de plus que $f_{k}\left(\bar{\theta}_{k}\right)=0$.

Le coût de production par unité de surface est $c_{k}=\mu_{k} \theta+\hat{c}_{k}$ dans l'expression duquel $\mu_{k}$ et $\hat{c}_{k}$ sont deux paramètres qui ne dépendent que du pays $k$. La demande intérieure est une fonction $\bar{y}_{k}(p)$ du prix européen de transaction intérieure $p$. La demande agrégée est $\bar{Y}(p)=\sum_{k \in \mathcal{K}} \bar{y}_{k}(p)$. Le 
coût d'opportunité sociale des fonds publics, noté $1+\lambda$ (avec $\lambda \geqslant 0$ ), est commun à tous les États-membres, traduisant la convergence des économies de ces États. On introduit une préférence sociale différenciée par pays pour les agriculteurs $1+\alpha_{k}$ telle que $0 \leqslant \alpha_{k} \leqslant \lambda$. Le prix mondial $e$ est invariant par rapport à l'offre domestique sur le marché mondial (hypothèse du « petit pays ").

Pour simplifier les expressions à venir, on notera $\hat{p}_{k}$ et $\hat{e}_{k}$ les termes dépendant de l'indice du pays par lesquels interviendront les prix:

$$
\begin{aligned}
& \hat{p}_{k}=p-\mu_{k} \\
& \hat{e}_{k}=e-\mu_{k}
\end{aligned}
$$

Et on fera l'hypothèse qu'il existe, dans chacun des pays concernés, des entreprises produisant au prix mondial en dégageant une marge positive:

$$
\forall k: \frac{\hat{c}_{k}}{\hat{e}_{k}}<\bar{\theta}_{k}
$$

la marge brute d'une unité de surface en production au prix mondial s'écrivant $\hat{e}_{k} \theta-\hat{c}_{k}$.

La politique est ajustée en fonction du rendement de chacune des firmes, de sorte que la politique proposée par l'autorité du pays $k$ à la firme $\theta$ consiste en l'offre d'une prime $t_{k}(\theta)$ en échange du retrait des terres à un taux $s_{k}(\theta)\left(s_{k} \in[0,1]\right)$. L'intervention publique concerne toutes les firmes en état de produire au prix garanti $p$ associé à la politique de gel, c'est-à-dire toutes les firmes dont le rendement est supérieur à $\hat{c}_{k} / \hat{p}_{k}$. Elle concerne également les firmes présentes dans la situation «hors gel " de référence et qui pourraient être exclues du marché par des prix trop bas. La situation de référence est rapportée à l'absence d'incitation au retrait des terres. La politique agricole est alors identifiée à une intervention publique via le système des restitutions à l'exportation associé à une politique de prix garanti de niveau $p_{0}$. Le prix de référence détermine par hypoyhèse l'ensemble des entreprises présentes dans le jeu économique, avec ou sans contrat:

$$
\forall k: \underline{\theta}_{k}=\frac{\hat{c}_{k}}{\hat{p}_{0 k}}
$$

Nous précisons maintenant les éléments du contrat dans un contexte d'autosélection ${ }^{1}$. Du fait de l'asymétrie d'information, le Principal est obligé d'intégrer dans son programme de régulation économique les firmes normalement exclues de la production après changement du prix garanti $\left(\frac{\hat{c}_{k}}{\hat{p}_{k}} \geqslant \underline{\theta}_{k}\right)$.

\footnotetext{
1 Nous ne retenons ici que les contrats librement consentis et acceptés dans un contexte d'information imparfaite. On trouvera des analyses des politiques de gel de terre obligatoire dans différents contextes informattonnels dans d'autres articles, en particulier Bourgeon et al., 1995, Jayet, 2001).
} 
En application du principe de révélation, le mécanisme incitatif est constitué d'un couple $\left(s_{k}(\tilde{\theta}), t_{k}(\tilde{\theta})\right)$ de taux de retrait $s_{k}$ et de transfert $t_{k}$, lorsque la firme annonce $\tilde{\theta}$ comme caractéristique.

Nous noterons $B_{k}$ l'ensemble des $\theta$ auxquels il est proposé un contrat. Nous utiliserons la notation $1_{X}(x)$ pour désigner la fonction indicatrice de $x$ sur l'ensemble $X$ (à valeur 1 sur $X, 0$ sinon). Par hypothèse, $B_{k} \subseteq\left[\underline{\theta}_{k}, \bar{\theta}_{k}\right]$.

Le profit de la firme $\theta$ qui annonce $\tilde{\theta}$ s'écrit :

$$
\tilde{\pi}_{k}(\theta, \tilde{\theta})=\left(\hat{p}_{k} \theta-\hat{c}_{k}\right)\left(1-s_{k}(\tilde{\theta})\right) \prod_{\theta \geqslant \hat{c}_{k} / \hat{p}_{k}}(\theta)+t_{k}(\tilde{\theta})
$$

L'autorité publique doit intégrer dans son programme une contrainte de rationalité, qui exprime l'idée que la firme à qui elle souhaite proposer un contrat ait effectivement intérêt à y participer. Le profit de la firme « régulée » doit alors être au moins égal au profit obtenu en l'absence d'engagement sur le retrait des terres. Tenant compte du fait que l'entreprise ne produit que si sa marge par unité de surface est positive, la contrainte de participation s'écrit :

$$
t_{k}(\theta) \geqslant\left(\hat{p}_{k} \theta-\hat{c}_{k}\right) s_{k}(\theta) \prod_{\theta \geqslant \hat{c}_{k} / \hat{p}_{k}}(\theta)
$$

Autrement dit, la rente d'information d'une entreprise contractante est positive.

Par ailleurs, il s'agit d'inciter la firme à choisir le contrat que le Principal souhaite qu'elle choisisse, en application du principe de révélation. Le contrat proposé aux firmes étant fonction de l'annonce par la firme de sa caractéristique, ce principe établit qu'il n'y a pas de meilleur mécanisme incitatif que les mécanismes directs révélateurs. En d'autres termes, compte tenu du barème proposé par le Principal, la firme ne doit pas avoir d'autre choix que d'annoncer sa véritable caractéristique. On trouvera dans Jayet (2001)la justification des contraintes d'incitation résultant du programme de maximisation du profit régulê par la firme. Ces conditions sont les suivantes $^{2}$ :

$$
\begin{gathered}
\dot{t}_{k}(\theta)=\left(\hat{p}_{k} \theta-\hat{c}_{k}\right) \dot{s}_{k}(\theta) \\
\dot{s}_{k}(\theta) \leqslant 0
\end{gathered}
$$

La rente $R_{k}(\theta)=t_{k}(\theta)-\left(\hat{p}_{k} \theta-\hat{c}_{k}\right) s_{k}(\theta) \prod_{\theta \geqslant \hat{c}_{k} / \hat{p}_{k}}(\theta)$ étant une fonction décroissante de $\theta$ si le gel est effectif, (plus précisément $\dot{R}_{k}(\theta)=-\hat{p}_{k} s_{k}(\theta)<$ 0 si $s_{k}(\theta)>0$ ), les firmes qui peuvent être intéressées par le contrat de gel ne peuvent être que des firmes de rendement inférieur au rendement pivot $\theta_{k}$ qui annule la rente $\left(\theta_{k} \leqslant \bar{\theta}_{k}\right)$. Toute firme contractante de rendement inférieur à $\theta_{k}$ bénéficiera d'une rente positive. Par ailleurs, supposons que l'autorité de régulation propose le contrat à une firme de rendement $\theta$. Il

2 On notera $\dot{x}$ la différentielle première d'une variable $x$ par rapport à $\theta$. Rappelons que la décroissance de la rente d'information, démontrée par ailleurs, justifie que l'on s'intéresse à des mécanismes différentiables. 
est aisé de vérifier que si l'autorité ne retient pas dans les firmes éligibles au contrat une firme de rendement $\theta^{\prime}$ tel que $\theta^{\prime}<\theta$, alors il est de l'intérêt de cette firme de se « faire passer » pour la firme $\theta$. L'ensemble $B_{k}$ est donc un intervalle de la forme:

$$
B_{k}=\left[\underline{\theta}_{k}, \theta_{k}\right]
$$

Les deux conditions (7) et (8) impliquent que le transfert maximal offert sera nécessairement égal à $t_{k}\left(\hat{c}_{k} / \hat{p}_{k}\right)=t_{k}\left(\underline{\theta}_{k}\right)$, transfert dont bénéficieront les firmes éventuellement exclues du marché. Ces firmes gèleront toutes leurs terres même sans y être contractuellement obligées, de sorte que $s_{k}(\theta)=1$ si $\theta \leqslant \hat{c}_{k} / \hat{p}_{k}$. Si les conditions d'incitation sont vérifiées pour $\theta \leqslant \hat{c}_{k} / \hat{p}_{k}$, elles sont donc vérifiées pour tout $\theta$.

Notons $\pi_{k}(\theta)$ le profit de la firme régulée $\left(\pi_{k}(\theta)=\left(\hat{p}_{k} \theta-\hat{c}_{k}\right)(1-\right.$ $\left.\left.s_{k}(\theta)\right)+t_{k}(\theta)\right)$.

\section{Subsidiarité en contrats}

On considère que le bien-être social à l'échelle du pays $k$ est la somme de la variation de surplus des consommateurs (définie relativement à un niveau de prix $p$ ) et des profits des producteurs pondérés par la préférence sociale $\alpha_{k}$, diminuée du coût social de l'intervention publique. Le contribuable du pays $k$ est sollicité au titre des restitutions aux exportations et au titre du paiement des transferts. Les transferts sont supportés par les budgets nationaux tandis que le coût des restitutions aux exportations est pris en charge par le budget européen auquel chaque pays participe à hauteur du taux $\beta_{k}$. Le surplus du consommateur est normé à 0 au prix de référence, de sorte que, faisant apparaître successivement le surplus du consommateur, le profit des producteurs, le coût des restitutions aux exportations et le coût des transferts consécutifs aux contrats, le niveau de bien-être social s'écrit :

$$
\begin{aligned}
W_{k} & =-\int_{p_{0}}^{p} \bar{y}_{k}(u) d u+\left(1+\alpha_{k}\right) S_{k} \int_{\underline{\theta}_{k}}^{\bar{\theta}_{k}} \pi_{k}(\theta) f_{k}(\theta) d \theta \\
& -(1+\lambda) \beta_{k}(p-e)\left(\sum_{k^{\prime} \in \mathcal{K}} S_{k^{\prime}} \int_{\underline{\theta}_{k^{\prime}}}^{\bar{\theta}_{k^{\prime}}} \theta\left(1-s_{k^{\prime}}(\theta)\right) f_{k^{\prime}}(\theta) d \theta-\bar{Y}(p)\right) \\
& -(1+\lambda) S_{k} \int_{\underline{\theta}_{k}}^{\bar{\theta}_{k}} t_{k}(\theta) f_{k}(\theta) d \theta
\end{aligned}
$$

En regroupant les termes affectant le contrat d'un pays $k$ (producteur), cette expression peut être réécrite sous la forme:

$W_{k}=-\int_{p_{0}}^{p} \bar{y}_{k}(u) d u+(1+\lambda) \beta_{k}(p-e) \bar{Y}(p)$ 


$$
\begin{aligned}
& -(1+\lambda) \beta_{k}(p-e) \sum_{\substack{k^{\prime} \in \mathcal{K} \\
k^{\prime} \neq k}} S_{k^{\prime}} \int_{\underline{\theta}_{k^{\prime}}}^{\bar{\theta}_{k^{\prime}}} \theta\left(1-s_{k^{\prime}}(\theta)\right) f_{k^{\prime}}(\theta) d \theta \\
& +S_{k} \int_{\underline{\theta}_{k}}^{\bar{\theta}_{k}}\left(\left(1+\alpha_{k}\right)\left(\hat{p}_{k} \theta-\hat{c}_{k}\right)-(1+\lambda) \beta_{k}(p-e) \theta\right) f_{k}(\theta) d \theta \\
& +S_{k} \int_{\underline{\theta}_{k}}^{\bar{\theta}_{k}}\left[\left((1+\lambda) \beta_{k}(p-e) \theta-\left(1+\alpha_{k}\right)\left(\hat{p}_{k} \theta-\hat{c}_{k}\right)\right) s_{k}(\theta)-\left(\lambda-\alpha_{k}\right) t_{k}(\theta)\right]
\end{aligned}
$$

Les paramètres du contrat pour le pays producteur $k$ n'apparaissent que dans la dernière intégrale que l'on peut réécrire uniquement en fonction de $s_{k}(\theta)$ après avoir intégré par parties $\int_{\underline{o}_{k}}^{\bar{o}_{k}} t_{k}(\theta) f_{k}(\theta) d \theta$, ce qui permet d'exprimer le bien-être social sous la forme (voir annexe) :

$$
W_{k}=\text { Constante }-S_{k} \int_{\underline{\theta}_{k}}^{\bar{\theta}_{k}} h_{k}(\theta) s_{k}(\theta) d \theta
$$

où la fonction $h_{k}(\theta)$ est définie par :

$$
h_{k}(\theta)=(1+\lambda)\left(\left(1-\beta_{k}\right) \hat{p}_{k} \theta+\beta_{k} \hat{e}_{k} \theta-\hat{c}_{k}\right) f_{k}(\theta)+\left(\lambda-\alpha_{k}\right) \hat{p}_{k} F_{k}(\theta)
$$

Compte tenu des hypothèses faites, $\left(F_{k}\right.$ de classe $\mathcal{C}^{2}: \lambda-\alpha_{k}>0$, $\left.f_{k}\left(\bar{\theta}_{k}\right)=0\right)$, la fonction $h_{k}(\theta)$ est continue sur $\left[\underline{\theta}_{k}, \bar{\theta}_{k}\right]$, à valeur négative en $\underline{\theta}_{k}$ et positive en $\bar{\theta}_{k}$. Elle s'annule donc au moins une fois sur l'intervalle. Par ailleurs, comme $\underline{\theta}_{k}<\frac{\hat{c}_{k}}{\hat{p}_{k}}<\frac{\hat{c}_{k}}{\left(1-\beta_{k}\right) \hat{p}_{k}+\beta_{k} \hat{e}_{k}}<\frac{\hat{c}_{k}}{\hat{e}_{k}}<\bar{\theta}_{k}$, et $f$ étant de classe $\mathcal{C}^{1}$; on a $h_{k}^{\prime}\left(\frac{\hat{c}_{k}}{\left(1-\beta_{k}\right) \hat{p}_{k}+\beta_{k} \hat{\varepsilon}_{k}}\right)>0$. En calculant la dérivée $h_{k}^{\prime}(\theta)$ en une solution $\theta_{s}$; la condition $h_{k}^{\prime}\left(\theta_{s}\right)>0$ équivaut à $\frac{d}{d \theta}\left(\frac{F(\theta)}{f(\theta)}\right)>-\frac{(1+\lambda)}{\left(\lambda-\alpha_{k}\right)} \frac{\left(1-\beta_{k}\right) \tilde{p}_{k}+\beta_{k} \hat{e}_{k}}{\hat{p}_{k}}{ }^{3} \mathrm{Si}$ cette dernière condition est vérifiée alors il y a unicité de la solution. C'est ce qu'on supposera par la suite. On peut maintenant décrire la politique optimale :

Proposition 1. Soit $\theta_{k}$ la solution de l'équation $h_{k}(\theta)=0$ dont on supposera qu'elle vérifie $\theta_{k}>\hat{c}_{k} / \hat{p}_{k}$. Si une politique de gel doit être instaurée, à prix $p$ donné, elle est telle que les firmes de caractéristique inférieure au seuil $\theta_{k}$, et seulement celles-ci, gèlent leurs terres (i.e. $s_{k}(\theta)=1$ ) en contrepartie d'une prime constante $t_{k}(\theta)=\hat{p}_{k} \theta_{k}-\hat{c}_{k}$.

Preuve. La démonstration découle immédiatement de l'expression $\mathrm{du}$ critère public et de la définition de la fonction $h_{k}$. Sous les hypothèses faites,

${ }^{3}$ On notera qu'ici, l'hypothèse standard de croissance croissant n'est pas suffisante à assurer que la fonction $h$ soit positive en $\bar{\theta}$. 
la fonction $h_{k}(\theta)$ est négative si $\theta \in\left[\underline{\theta}_{k}, \theta_{k}\right]$ et positive si $\in\left[\theta_{k}, \bar{\theta}_{k}\right]$. La solution $\theta_{k}$ de l'équation $h_{k}(\theta)=0$ caractérise donc le rendement seuil tel que $B_{k}=\left[\underline{\theta}_{k}, \theta_{k}\right]$. La contrainte d'incitation (7) et la contrainte de participation (6) impliquent que la prime est constante et vaut $t_{k}(\theta)=\hat{p}_{k} \theta_{k}-\hat{c}_{k}$.

Remarque 1. On étendra la notion de rendement seuil aux pays producteurs qui n'ont pas intérêt à proposer de contrat de gel, de sorte que $\theta_{k}=\hat{c}_{k} / \hat{p}_{k}$ s'il n'existe pas de solution à l'équation $h_{k}(\theta)=0$ qui soit supérieure à $\hat{c}_{k} / \hat{p}_{k}$.

À l'équilibre de Nash en contrats, chaque État détermine le contrat optimal de gel de terre qu'il offre à ses producteurs, les autres États agissant de même. Le bien-être social est alors, lorsque joue la subsidiarité :

$$
\begin{aligned}
W_{k}^{N} & =-\int_{p_{0}}^{p} \bar{y}_{k}(u) d u+(1+\lambda) \beta_{k}(p-e) \bar{Y}(p) \\
& -(1+\lambda) \beta_{k}(p-e) \sum_{\substack{k^{\prime} \in \mathcal{K} \\
k^{\prime} \neq k}} S_{k^{\prime}} \int_{\underline{\theta}_{k^{\prime}}}^{\bar{\theta}_{k^{\prime}}} \theta f_{k^{\prime}}(\theta) d \theta \\
& +S_{k} \int_{\underline{\theta}_{k}}^{\bar{\theta}_{k}}\left[\left(1+\alpha_{k}\right)\left(\hat{p}_{k} \theta-\hat{c}_{k}\right)-(1+\lambda) \beta_{k}(p-e) \theta\right] f_{k}(\theta) d \theta \\
& -S_{k} \int_{\underline{\theta}_{k}}^{\theta_{k}} h_{k}(\theta) d \theta
\end{aligned}
$$

\section{Contrat unique européen}

On suppose que les préférences sociales telles que les reconnaissent les Étatsmembres vis à vis de leurs producteurs sont reconnues avec les mêmes valeurs à l'échelle des instances communautaires. Le contrat de gel est ici unique $\left(s_{U E}(\theta), t_{U E}(\theta)\right)$. La fonction de bien-être totale de l'UE est alors :

$$
\begin{aligned}
W_{U E} & =-\int_{p_{0}}^{p} \bar{Y}(u) d u+\sum_{k \in \mathcal{K}}\left(1+\alpha_{k}\right) S_{k} \int_{\underline{Q}_{k}}^{\bar{\theta}_{k}} \pi_{k}(\theta) f_{k}(\theta) d \theta \\
& -(1+\lambda)(p-e)\left(\sum_{k \in \mathcal{K}} S_{k} \int_{\underline{\theta}_{k}}^{\bar{\theta}_{k}} \theta\left(1-s_{U E}(\theta)\right) f_{k}(\theta) d \theta-\bar{Y}(p)\right) \\
& -(1+\lambda) \sum_{k \in \mathcal{K}} S_{k} \int_{\underline{\theta}_{k}}^{\bar{\theta}_{k}} t_{U E}(\theta) f_{k}(\theta) d \theta
\end{aligned}
$$

que l'on peut réécrire sous la forme

$$
W_{U E}=-\int_{p_{0}}^{p} \bar{Y}(u) d u+(1+\lambda)(p-e) \bar{Y}(p)
$$




$$
\begin{aligned}
& -\sum_{k \in \mathcal{K}} S_{k} \int_{\underline{\theta}_{k}}^{\bar{\theta}_{k}}\left[(1+\lambda)(p-e) \theta-\left(1+\alpha_{k}\right)\left(\hat{p}_{k} \theta-\hat{c}_{k}\right)\right] f_{k}(\theta) d \theta \\
& +\sum_{k \in \mathcal{K}} S_{k} \int_{\underline{\theta}_{k}}^{\bar{\theta}_{k}}\left[(1+\lambda)(p-e) \theta-\left(1+\alpha_{k}\right)\left(\hat{p}_{k} \theta-\hat{c}_{k}\right)\right] s_{U E}(\theta) f_{k}(\theta) d \theta \\
& -\sum_{k \in \mathcal{K}} S_{k} \int_{\underline{\theta}_{k}}^{\bar{\theta}_{k}}\left(\lambda-\alpha_{k}\right) t_{U^{\prime} E}(\theta) f_{k}(\theta) d \theta
\end{aligned}
$$

Le problème du contrat supposé unique à l'échelle européenne est analogue au problème résolu à l'échelle nationale. On en déduit en particulier que le gel concernera les firmes de rendement inférieur à un seuil unique que nous noterons $\theta_{U E}$, de sorte que $B_{k}=\left[\underline{\theta}_{k}, \theta_{U E}\right]$. De plus, la contrainte d'incitation $\dot{t}_{U E}(\theta)=\left(\hat{p}_{k} \theta-\hat{c}_{k}\right) \dot{s}_{U E}(\theta)$ est. intégrée sur l'ensemble des exploitations agricoles de l'UE de la même manière que pour un pays $k$ dans le cas de la subsidiarité, et on a :

$$
\begin{aligned}
& \int_{\underline{\theta}_{k}}^{\bar{\theta}_{k}} t_{U E}(\theta) f_{k}(\theta) d \theta=\left[t_{U E}\left(\bar{\theta}_{k}\right)-\left(\hat{p}_{k} \bar{\theta}_{k}-\hat{c}_{k}\right) s_{U E}\left(\bar{\theta}_{k}\right)\right] \\
&+\int_{\underline{\theta}_{k}}^{\bar{\theta}_{k}}\left(\left(\hat{p}_{k} \theta-\hat{c}_{k}\right) f_{k}(\theta)+\hat{p}_{k} F_{k}(\theta)\right) s_{U E}(\theta) d \theta \\
& \forall k \text { tel que } S_{k}>0
\end{aligned}
$$

En sommant sur $k$ et en substituant l'expression du transfert dans l'expression de la fonction de bien-être tout en tenant compte du fait que seules les firmes des pays pour lesquelles $\theta \leqslant \theta_{U E}$ sont régulées, on obtient ${ }^{4}$ :

$$
\begin{aligned}
W_{\ell E E} & =-\int_{p_{0}}^{p} \bar{Y}(u) d u+(1+\lambda)(p-e) \bar{Y}(p) \\
& -\sum_{k \in \mathcal{K}} S_{k} \int_{\underline{\theta}_{k}}^{\ddot{\theta}_{k}}\left[(1+\lambda)(p-e) \theta-\left(1+\alpha_{k}\right)\left(\hat{p}_{k} \theta-\hat{c}_{k}\right)\right] f_{k}(\theta) d \theta \\
& -\sum_{k \in \mathcal{K}} S_{k} \int_{\underline{\theta}_{k}}^{\theta_{t^{\prime}}}\left[(1+\lambda)\left(\hat{e}_{k} \theta-\hat{c}_{k}\right) f_{k}(\theta)+\left(\lambda-\alpha_{k}\right) \hat{p}_{k} F_{k}(\theta)\right] d \theta \\
& -\sum_{k \in \mathcal{K}} S_{k}\left(\lambda-\alpha_{k}\right)\left[t_{\ell: E}\left(\bar{\theta}_{k}\right)-\left(\hat{p}_{k} \bar{\theta}_{k}-\hat{c}_{k}\right) s_{U_{E} E}\left(\bar{\theta}_{k}\right)\right]
\end{aligned}
$$

On s'intéresse au cas où les pays, quelle que soit la politique communautaire de gel, restent partiellement producteurs, au sens où aucun pays n'est contraint à tout geler. Autrement dit, on suppose dans la suite

\footnotetext{
${ }^{4}$ On remarquera qu'ici, ce dernier crochet n'est pas nécessairement nul car il est possible que pour certains pays $k$ on ait $\vec{\theta}_{k}<\theta_{U} k$.
} 
$\theta_{U E}<\bar{\theta}_{k}$ quel que soit $k$. Dans ces conditions, on a $s_{U E}\left(\bar{\theta}_{k}\right)=0$ et donc $t_{U E}\left(\bar{\theta}_{k}\right)=0$. Il s'ensuit que le dernier terme de l'expression précédente donnant $W_{U E}$ est nul.

La forme de ce critère est la même que celle étudiée dans la section précédente et la valeur seuil $\theta_{U E}$ est définie ici comme la solution de l'équation $H(\theta)=0$, où la fonction de commutation est donnée par :

$$
H(\theta)=\sum_{k \in \mathcal{K}} S_{k}\left[(1+\lambda)\left(\hat{e}_{k} \theta-\hat{c}_{k}\right) f_{k}(\theta)+\left(\lambda-\alpha_{k}\right) \hat{p}_{k} F_{k}(\theta)\right]
$$

\section{Comparaison des deux types de contrats}

Comme nous le verrons, la comparaison des politiques de gel de terres ne permettra pas de mettre en évidence un avantage communautaire de l'une par rapport à l'autre. Rappelons que les points communs de ces politiques sont la mise en oeuvre de contrats librement acceptés par les producteurs, dans un cadre d'asymétrie d'information rendant les Etats membres et la Commission Européenne non informés sur les caractéristiques individuelles de producteurs. Les politiques ne diffèrent que par les modalités d'application. Dans un cas, les États membres sont libres de proposer simultanément les contrats qu'ils proposent à leurs producteurs nationaux. Dans l'autre, les États laissent la Commission proposer un seul type de contrat à l'ensemble des producteurs de l'Union Européenne. Nous proposons d'aborder ce problème dans le cas le plus simple où le prix garanti $p$ est identique.

Pour comparer les deux types de politiques, le prix $p$ étant fixé, il nous faut calculer la différence des bien-êtres qu'elles permettent d'atteindre. On montre en annexe que cette différence est égale à

$$
\begin{aligned}
W_{U E}-W^{N} & =\sum_{k \in \mathcal{K}} S_{k}\left\{(1+\lambda)(p-e)\left(1-\beta_{k}\right) \int_{\underline{\theta}_{k}}^{\theta_{k}} \theta f_{k}(\theta) d \theta\right. \\
& \left.+\int_{\theta_{U E}}^{\theta_{k}}\left[(1+\lambda)\left(\hat{e}_{k} \theta-\hat{c}_{k}\right) f_{k}(\theta)+\left(\lambda-\alpha_{k}\right) \hat{p}_{k} F_{k}(\theta)\right]\right\} d \theta
\end{aligned}
$$

Il est a priori difficile de comparer les bien-êtres sociaux totaux obtenus selon les deux types de politiques, compte tenu des nombreux paramètres qui interviennent dans leur détermination. Aussi, pour fixer les idées, nous examinons une situation limite où les pays sont tous identiques, à l'exception des surfaces cultivées. Autrement dit nous considérons un cas où $\hat{p}_{k}=\hat{p}, f_{k}=f, \hat{c}_{k}=\hat{c}, \hat{e}_{k}=\hat{e}, \alpha_{k}=\alpha, \bar{\theta}_{k}=\bar{\theta}, \underline{\theta}_{k}=\underline{\theta}$, et donc $\beta_{k}=\beta=\frac{1}{K}$ où $K$ est le nombre de pays producteurs. 
Dans ces conditions, l'expression précédente devient

$$
\begin{aligned}
W_{L^{\prime} E}-W^{N} & =\sum_{k \in \mathcal{K}} S_{k}\left\{(1+\lambda)(p-e)\left(1-\frac{1}{K}\right) \int_{\underline{0}}^{\theta^{*}} \theta f(\theta) d \theta\right. \\
& +\int_{\theta_{\ell E}}^{\theta^{*}}[(1+\lambda)(\hat{e} \theta-\hat{c}) f(\theta)+(\lambda-\alpha) \hat{p} F(\theta)] d \theta
\end{aligned}
$$

où $\theta^{*}$ est la valeur du pivot dans le contrat en subsidiarité.

Dans cette expression, le premier terme du second membre est positif. Il reste donc à définir le signe du deuxième terme du second membre. $\theta^{*}$ est défini comme la solution de l'équation :

$$
h(\theta, \beta)=(1+\lambda)[(1-\beta) \hat{p} \theta+\beta \hat{e} \theta-\hat{c}] f(\theta)+(\lambda-\alpha) \hat{p} F(\theta)=0
$$

tandis que $\theta_{U^{\prime} E}$ est solution de l'équation $h(\theta, 1)=0$

La fonction $h(\theta, \beta)$ est une fonction décroissante de $\beta$, par conséquent $h\left(\theta^{*}, 1\right)<h\left(\theta^{*}, \beta\right)=0=h\left(\theta_{V E}, 1\right)$. Sous les hypothèses faites dans la proposition 1 , la fonction $h(\theta, 1)$ est négative pour $\theta<\theta_{l i E}$ et positive pour $\theta>\theta_{U E}$. On en déduit donc immédiatement que $\theta^{*}<\theta_{U E}$.

Par conséquent, on a :

$$
\int_{\theta_{U E}}^{\theta^{-}}[(1+\lambda)(\hat{e} \theta-\hat{c}) f(\theta)+(\lambda-\alpha) \hat{p} F(\theta)] d \theta=-\int_{\theta^{*}}^{\theta_{U E}} h(\theta, 1) d \theta>0
$$

puisque $h(\theta, 1)$ est, négative pour $\theta<\theta_{U E}$. Il s'ensuit donc que $W_{U E}>W^{N}$. Par continuité, il en est de même quand les pays ont des paramètres proches. On peut alors énoncer la proposition suivante :

Proposition 2. À prix $p$ fixé, le bien-être social du contrat unique européen pour des pays présentant des caractéristiques proches, en termes de rendements, de coût de production unitaire et de participation au budget communautaire, est supérieur à la somme des biens-être nationaux à l'équilibre de Nash en contrats.

L'intuition de ce résultat vient de ce que, si les pays sont identiques concernant les paramètres qui jouent un rôle dans la détermination des contrats optimaux, ils vont individuellement choisir le même contrat optimal pour leurs producteurs. À l'équilibre de Nash, il y a donc un contrat unique, mais ce contrat ne tient pas compte des interdépendances entre pays, ce qui réduit l'ensemble des contrats admissibles, au contraire du contrat unique européen. Dès lors, étant défini dans un domaine plus large, ce dernier conduit nécessairement à un bien-être au moins égal à celui permis à l'équilibre de Nash. 


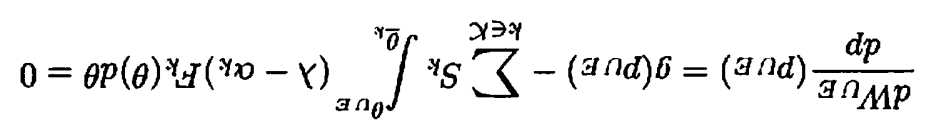

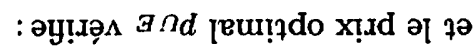

(72)

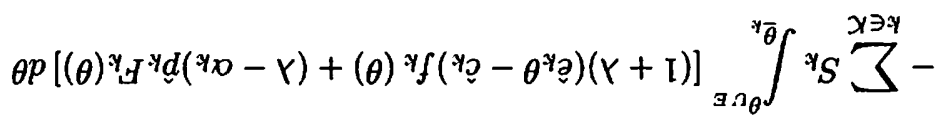

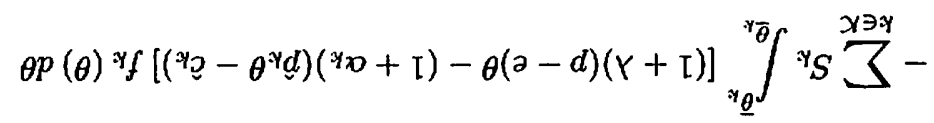

$$
\begin{aligned}
& (d) \underline{\Lambda}(\partial-d)(Y+I)+n p(n) \underline{\Lambda}_{d}{ }^{o d}-=B \Omega M
\end{aligned}
$$

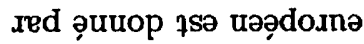

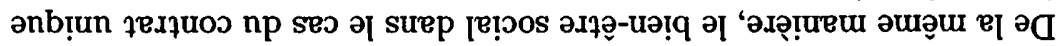

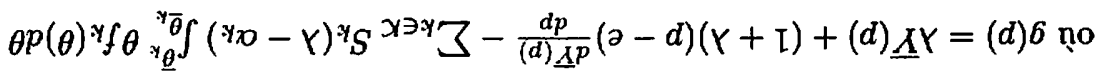

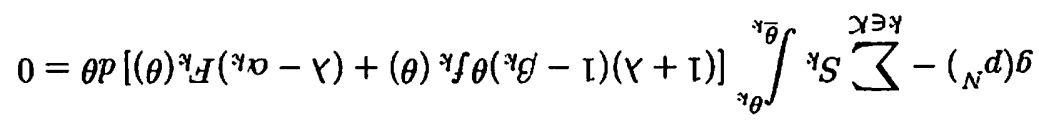

$$
\begin{aligned}
& =\left({ }_{N} d\right) \frac{d p}{{ }_{N} M P}
\end{aligned}
$$

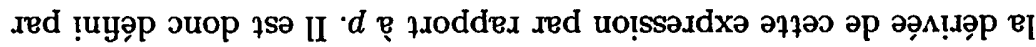

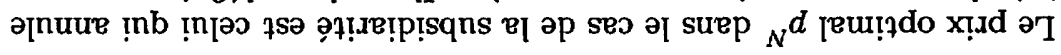

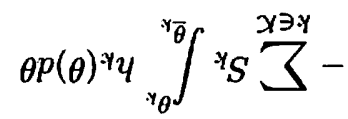

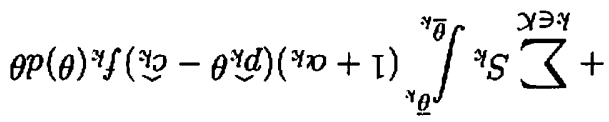

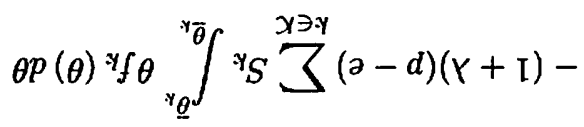

$$
\begin{aligned}
& (d)_{\underline{\Lambda}}(a-d)(\gamma+\mathrm{I})+n p(n) \underline{\Lambda}_{d} \int^{\mathrm{Dd}}-={ }_{N}^{y} M \stackrel{y}{\longrightarrow}={ }_{N} M
\end{aligned}
$$

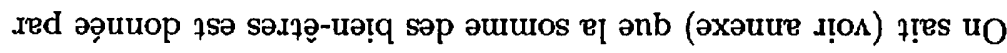

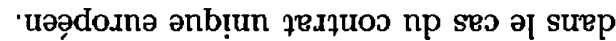

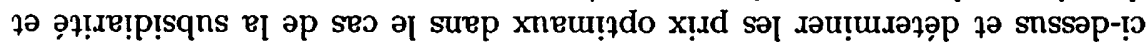

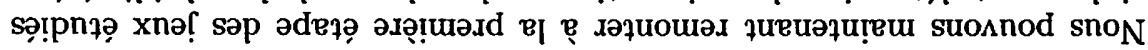


On sait d'après la section précédente, que quel que soit $p$ et dès que les pays producteurs présentent des caractéristiques communes en matière de coût, de distribution des rendements et de participation au budget communautaires, on a $W_{U E}>W^{N}$. Toutefois, on a généralement $p_{U E}$ différent de $p^{N}$. La comparaison de ces deux prix dans le cas général est difficile et il est probable que tous les cas sont possibles en fonction de la valeur des différents paramètres, aussi allons nous nous restreindre ici au cas symétrique déjà évoqué ci-dessus. Dans ces conditions, on obtient

$g\left(p^{N}\right)-g\left(p_{U E}\right)=\sum_{k \in \mathcal{K}} S_{k}\left\{\int_{\underline{\theta}}^{\theta^{*}}(1+\lambda)(1-\beta) \theta f(\theta) d \theta+\int_{\theta_{U E}}^{\theta^{*}}(\lambda-\alpha) F(\theta) d \theta\right\}$

Toutefois, même dans ce cas simple, il est impossible de conclure puisque la première intégrale dans l'accolade est positive alors que la seconde est négative puisque $\theta^{*}<\theta_{U E}$ et $\lambda-\alpha>0$. Ce n'est que si $\lambda=\alpha$ (où du moins $\lambda-\alpha$ négligeable), que l'on peut arriver à une conclusion. On a alors $g\left(p^{N}\right)-g\left(p_{U E}\right)>0$ et $p_{U^{\prime} E}>p^{N}$ puisque $\frac{d g}{d p}<0 .^{5}$

Dans ce cas très particulier, le bien-être social associé au contrat unique européen est maximum pour un prix supérieur à celui qui permet d'obtenir un bien-être maximum dans le cas de la subsidiarité. Toutefois le cadre théorique qui vient d'être développé permet de proposer une analyse empirique, dont nous donnons un exemple dans la section suivante.

\section{$7 \quad$ Application empirique}

Comme nous l'avons indiqué en introduction, différents domaines d'application sont envisageables. Compte tenu des données disponibles et des enjeux financiers à l'échelle européenne, nous proposons une application portant sur le secteur de production du blé tendre.

Les données utilisées proviennent de sources statistiques européennes (EUROSTAT et RICA) et américaines (USDA). Elles concernent les rendements, surfaces et charges variables pour 12 pays de l'Union en 1994 (hors Allemagne de l'Est). On dispose également des demandes de chacun des pays adressées aux pays producteurs de l'Union. Les contributions au budget européen (Fonds Européen d'Orientation et de Garantie Agricole FEOGA) correspondent aux contributions de l'Union à 12 qui prévalaient avant l'élargissement. Ce jeu de données est complété par des prix moyens garanti et mondial pour 1994 (respectivement 0.15 et 0.09 ECU94 $/ \mathrm{kg}$ ). L'élasticité prix propre de la demande est supposée identique pour tous les pays membres $(-0.3)$ au prix de référence. À ce prix, la demande européenne est

5 On a $\frac{d g}{d p}=(1+2 \lambda) \frac{d \bar{Y}}{d p}+(1+\lambda)(p-e) \frac{d^{2} \bar{Y}}{d p^{2}}<0$ pour une fonction de demande linéaire, concave, ou pas trop convexe en négligeant les eftets de second ordre. 
connue, ainsi que les parts nationales de cette demande européenne que l'on supposera indépendantes du prix.

On fait l'hypothèse de distributions log-normales des rendements de blé au sein de chacun des pays producteurs. L'ensemble des données caractérisant chacun des pays est présenté dans le tableau 1 en annexe 9.3. Moyennes, variances, et charges variables caractérisant les rendements sont issues d'estimations fondées sur les données micro-économiques du RICA européen.

Nos simulations reposent sur une valeur du coût d'opportunité des fonds publics fréquemment utilisée dans la littérature, égale à 1.4. Nous retiendrons l'hypothèse d'une valeur de la préférence sociale accordée aux producteurs commune aux différents États membres de l'Union. Elle est calculée en considérant que le prix garanti observé en l'absence de politique de retrait des terres résulte d'un choix social optimal dans le cadre d'une politique agricole combinant prix garanti et resitution aux exportations sur pays tiers. Elle s'obtient à partir de l'équation $g(p)=0$ résolue en $\alpha$ (voir section 6). Avec nos hypothèses, $\alpha=0.22$.

La résolution du système d'équations en prix et rendements seuils est obtenue à partir de programmes FORTRAN. Nous présentons en annexe successivement les rendements seuils et primes (tableau 2), puis les résultats économiques synthétisant les effets de la politique envisagée (tableaux $3.1-3)$. Précisons ici la valeur des prix dans les différentes configurations envisagées. Le prix garanti de la situation de référence (hors programme de gel) est de $0.150 \mathrm{kECU} 94 / \mathrm{t}$. Les prix dans les situations de gel subsidiaire et de gel européen valent respectivement 0.140 et 0.147 .

On peut dégager quelques observations des résultats numériques obtenus. Tout d'abord, la mise en oeuvre des programmes d'incitation au gel des terres se traduirait par un gel effectif, selon la forme théorique définie. Mais la traduction en terme de bien-être social paraît limitée, avec un gain social de l'ordre de 100 millions d'ECU pour l'Union Européenne. Ce résultat diffère quelque peu de résultats obtenus dans le cadre d'une politique de gel de terre « renationalisée », telle qu'elle avait été étudiée pour la France (Jayet, 2001). On peut l'expliquer par l'existence d'un effet « passager clandestin » lié à la mutualisation des coûts de la politique agricole.

Cet effet explique sans doute qu'une politique fondée sur la subsidiarité, et laissant aux États une marge de manoeuvre en théorie importante, est caractérisée par un très faible gel de terre, de 1 à $9 \%$ selon les États membres (tableau 2). Une politique de contrat uniforme ferait apparaître un contraste important entre les États, le gel affectant les pays aux coûts les plus élevés et aux rendements les plus faibles devenant significatif (supérieur à $20 \%$, à l'exception des Pays-Bas et du Royaume Uni). Les variations de bien-être et de ses composantes (producteurs, consommateurs, contribuables) sont données pour chaque pays et dans les différentes configurations de politique économique sur le tableau 3 . Les producteurs aux rendements les plus élevés (producteurs du «nord ») ont intérêt au statu quo. Il en est 
de même, en terme de welfare, pour le Danemark, la France et le Royaume Uni. La politique de gel subsidiaire sera préférée par les consommateurs et les contribuables de chacun des États membres, et, en terme collectif national, par 6 États membres (les États du Benelux, l'Allemagne et l'Italie). Enfin, le gel commun sera préféré par les producteurs du « sud » (espagnols, italiens, grecs, portugais) et, à l'échelle de l'Etat, par l'Espagne, l'Italie et le Portugal.

Il semble que la «domination sociale » d'une politique par rapport à l'autre dépende étroitement de la valeur des paramètres du problème. À titre d'exemple, pour les niveaux retenus des rendements et des charges variables, un fort niveau d'exportation sur pays tiers donnerait la préférence sociale à la politique de gel uniforme. Par contre, la politique de subsidiarité devrait être préférée à la politique de gel uniforme dans le cas d'exportations sur pays tiers d'un niveau plus faible et donc moins coûteuse en terme de restitutions aux exportations. Rappelons que les restitutions aux exportations sont supportées par le budget communautaire, à la différence des coûts des transferts consécutifs au gel subsidiaire qui sont supportés par les budgets nationaux. En résumé, le principal enseignement que l'on puisse retenir est que le choix de la politique n'est pas univoque et qu'aucune des deux politiques étudiées ne domine systématiquement l'autre.

On retiendra aussi, toujours dans le cadre de nos hypothèses numériques, que la variation de bien-être social entre les deux politiques est faible, et que l'accroissement du bien-être imputable au retrait des terres apparaît pour le moins limité (de l'ordre de quelques dizaines de millions d'Euros selon les différentes simulations réalisées). Par contre, les contrastes précédemment évoqués quant aux niveaux respectifs du gel et du montant des primes dans le cadre de l'une ou l'autre politique se traduisent par des variations de surplus significatives pour certains agents (de l'ordre de $10 \%$ des profits pour les producteurs français, et jusqu'à $50 \%$ des profits des producteurs espagnols contraints de geler la majorité des terres si la politique commune est adoptée).

\section{Conclusion}

La Politique Agricole Commune est en général reconnue comme étant inefficace, au sens où une politique moins coûteuse pour le contribuable et le consommateur pourrait être mise en oeuvre tout en étant compatible avec une préférence sociale avantageant le revenu agricole. Parmi les options souvent proposées, le gel de terres est l'un des moyens d'accroitre le bien-être social ( $\mathrm{au}$ sens où nous l'avons défini). L'un des problèmes qui est alors souvent posé est celui des modalités d'application des politiques proposées. Deux options s'affrontent à l'échelle communautaire. Tout en restant dans le cadre de la PAC et de ses principes (par exemple l'unicité des marchés et le soutien des revenus agricoles), la Commission Européenne peut proposer 
des modalités d'application nationales des politiques communes en application du principe de subsidiarité, ou au contraire laisser les États membres décider de ces modalités à condition d'en supporter tout ou partie des effets.

Cet article aborde ce type de choix pour les politiques de gel des terres en proposant des analyses théorique et numérique concernant l'une des principales organisation communes de marché, avec le blé tendre. L'application à d'autres produits, tels que les produits viticoles, peut conduire à des résultats sensiblement différents que ceux que nous avons présentés pour le blé. Le vin a l'avantage, du point de vue de la modélisation, d'être séparable des autres produits agricoles. Néanmoins, la difficulté de bien différencier les vins de table - soumis à régulation - des vins de qualité produits sur des régions déterminées (les v.q.p.r.d. recensés dans l'Union Européenne) rend plus délicat le traitement des données nécessaires aux calculs.

Néanmoins, à propos de la question importante traitant des décisions publiques à l'échelle de l'Union Européenne, deux résultats doivent être soulignés.

D'une part, en matière de politique de régulation des marchés agricoles via l'instrument du retrait des terres, laisser le choix des contrats de gel aux États membres ne garantit pas une meilleure efficacité qu'une politique de contrat unique de gel de terres proposée pour toute I'Union Européenne. Les premières simulations réalisées pour traiter le cas de l'OCM «blé » montrent à quel point, de la part du régulateur, une bonne appréciation des informations sur les caractéristiques du marché conditionne le niveau optimal de décision.

D'autre part, les intérêts divergents entre les différents acteurs (producteurs, consommateurs, contribuables) selon leur nationalité, et les intérêts divergents entre les États eux-mêmes, compliquent la décision publique finale. On peut trouver là une explication de la difficulté d'aboutir des « marathons agricoles » européens.

Enfin, compte tenu de la mutualisation à l'échelle européenne de la plus grande partie des dépenses publiques agricoles, et compte tenu du fait que les contributions budgétaires des États membres sont découplées des poids respectifs des économies nationales agricoles dans l'ensemble communautaire, l'accroissement de bien-être social n'apparaît pas très important. Ces éléments limitent les efforts que pourraient réaliser les États. Les gains n'en sont pas moins significatifs, mesurés ici pour un seul produit, le blé, en ne mettant en jeu qu'un mode de régulation, le gel de terre. Il y a donc encore matière à améliorer l'efficacité sociale de la PAC. 


\section{$9 \quad$ Annexes}

\subsection{Expression du bien-être social du pays $k$}

L'expression (11) permet d'écrire le bien-être social du pays $k$ sous la forme: $W_{k}=$ constante $+S_{k} \int_{\underline{\theta}_{k}}^{\vec{\theta}_{k}}\left[\left((1+\lambda) \beta_{k}(p-e) \theta-\left(1+\alpha_{k}\right)\left(\hat{p}_{k} \theta-\hat{c}_{k}\right)\right) s_{k}(\theta)-\left(\lambda-\alpha_{k}\right) t_{k}(\theta)\right] f_{k}(\theta) d \theta$

En intégrant par parties $\int_{\underline{\theta}_{k}}^{\bar{\theta}_{k}} t_{k}(\theta) f_{k}(\theta) d \theta$ il vient :

$$
\int_{\underline{\theta}_{k}}^{\bar{\theta}_{k}} t_{k}(\theta) f_{k}(\theta) d \theta=\left[t_{k}(\theta) F_{k}(\theta)\right]_{\underline{\theta}_{k}}^{\bar{\theta}_{k}}-\int_{\underline{\theta}_{k}}^{\bar{\theta}_{k}} \dot{t}_{k}(\theta) F_{k}(\theta) d \theta
$$

avec $\dot{t}_{k}(\theta)=\left(\hat{p}_{k} \theta-\hat{c}_{k}\right) \dot{s}_{k}(\theta)$

En intégrant de nouveau par parties la seconde intégrale, on a:

$$
\begin{aligned}
\int_{\underline{\theta}_{k}}^{\bar{\theta}_{k}}\left(\hat{p}_{k} \theta-\hat{c}_{k}\right) \dot{s}_{k}(\theta) F_{k}(\theta) d \theta & =\left[\left(\hat{p}_{k} \theta-\hat{c}_{k}\right) s_{k}(\theta) F_{k}(\theta)\right]_{\underline{\theta}_{k}}^{\bar{\theta}_{k}} \\
& -\int_{\underline{\theta}_{k}}^{\bar{\theta}_{k}}\left\{\left(\hat{p}_{k} \theta-\hat{c}_{k}\right) f_{k}(\theta)+\hat{p}_{k} F_{k}(\theta)\right\} s_{k}(\theta) d \theta
\end{aligned}
$$

Après substitution et en tenant compte du fait que $F_{k}\left(\underline{\theta}_{k}\right)=0$ et $F_{k}\left(\bar{\theta}_{k}\right)=1$, on obtient :

$$
\begin{aligned}
\int_{\underline{\theta}_{k}}^{\bar{\theta}_{k}} t_{k}(\theta) f_{k}(\theta) d \theta & =\left[t_{k}\left(\bar{\theta}_{k}\right)-\left(\hat{p}_{k} \bar{\theta}_{k}-\hat{c}_{k}\right) s_{k}\left(\bar{\theta}_{k}\right)\right] \\
& +\int_{\underline{\theta}_{k}}^{\bar{\theta}_{k}}\left\{\left(\hat{p}_{k} \theta-\hat{c}_{k}\right) f_{k}(\theta)+\hat{p}_{k} F_{k}(\theta)\right\} s_{k}(\theta) d \theta
\end{aligned}
$$

Dans cette dernière expression, le crochet est nul d'après la contrainte de participation et en remplaçant dans l'expression initiale on trouve finalement :

$$
\begin{aligned}
W_{k} & =\text { constante }-S_{k} \int_{\underline{\theta}_{k}}^{\bar{\theta}_{k}}\left\{(1+\lambda)\left(\left(1-\beta_{k}\right) \hat{p}_{k} \theta+\beta_{k} \hat{e}_{k} \theta-\hat{c}_{k}\right) f_{k}(\theta)\right. \\
& \left.+\left(\lambda-\alpha_{k}\right) \hat{p}_{k} F_{k}(\theta)\right\} s_{k}(\theta) d \theta \\
& =\text { constante }-S_{k} \int_{\underline{\theta}_{k}}^{\bar{\theta}_{k}} h_{k}(\theta) s_{k}(\theta) d \theta
\end{aligned}
$$




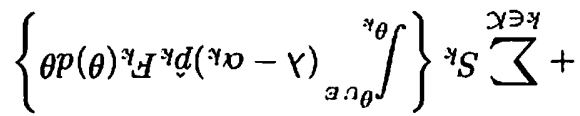

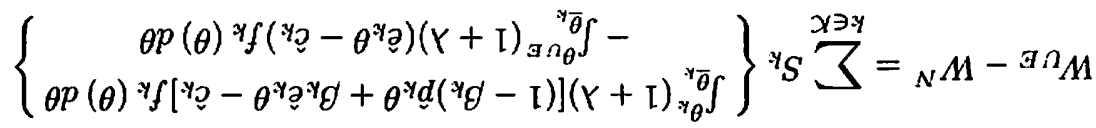

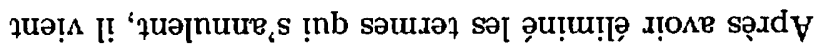

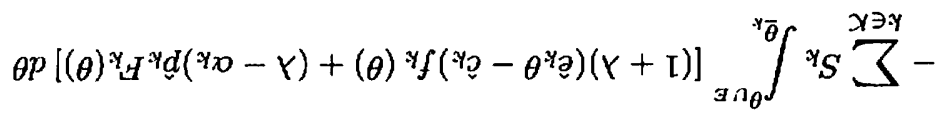

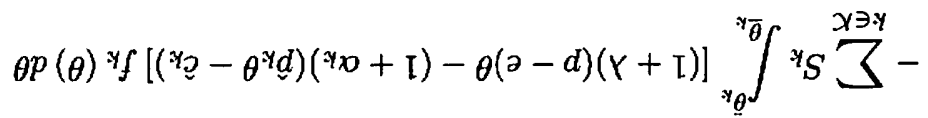

$$
\begin{aligned}
& (d) \underline{\Lambda}(\partial-d)(\gamma+\mathrm{I})+n p(n) \underline{\underline{X}} \int_{d}^{\mathrm{od}}-={ }^{3} \Omega_{M}
\end{aligned}
$$

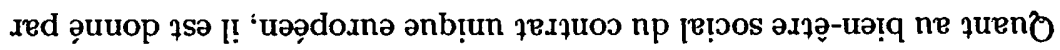

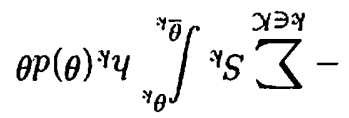

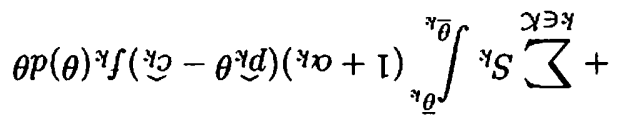

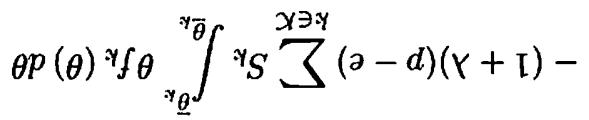

$$
\begin{aligned}
& (d) \underline{\Lambda}(\partial-d)(\gamma+\mathrm{I})+n p(n) \underline{\Lambda}{ }_{d}^{\mathrm{od}_{d}}-={ }_{N}^{y} M \stackrel{y}{\longrightarrow}
\end{aligned}
$$

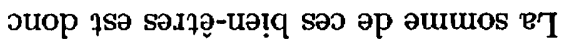

$$
\begin{aligned}
& \theta p(\theta)^{\gamma_{4}} \int_{{ }^{y} \theta}^{{ }^{*} \bar{\theta}}{ }^{y^{*}} S-
\end{aligned}
$$

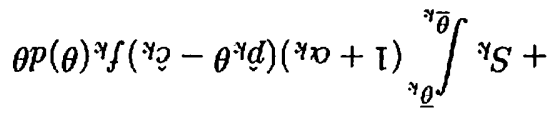

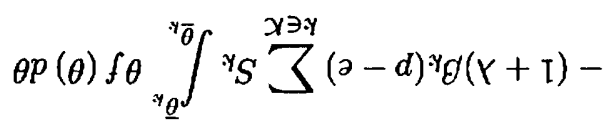

$$
\begin{aligned}
& (d)_{\underline{X}}(\partial-d)^{\gamma} g(Y+\tau)+n p(n)^{\gamma} \underline{\underline{\kappa}}{ }_{d}^{o d}-={ }_{N}^{\gamma} M
\end{aligned}
$$

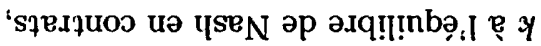

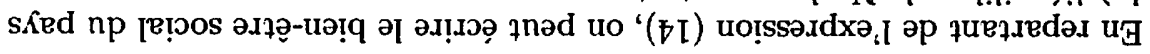

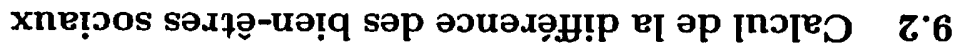


En remarquant que la première intégrale peut s'écrire

$$
\begin{aligned}
& \int_{\underline{\theta}_{k}}^{\theta_{k}}(1+\lambda)\left[\left(1-\beta_{k}\right) \hat{p}_{k} \theta+\beta_{k} \hat{e}_{k} \theta-\hat{e}_{k} \theta+\hat{e}_{k} \theta-\hat{c}_{k}\right] f_{k}(\theta) d \theta \\
& =\int_{\underline{\theta}_{k}}^{\theta_{k}}(1+\lambda)\left[\left(1-\beta_{k}\right) \hat{p}_{k} \theta+\left(\beta_{k}-1\right) \hat{e}_{k} \theta\right] f_{k}(\theta) d \theta \\
& +\int_{\underline{\theta}_{k}}^{\theta_{k}}(1+\lambda)\left(\hat{e}_{k} \theta-\hat{c}_{k}\right) f_{k}(\theta) d \theta
\end{aligned}
$$

On obtient finalement,

$$
\begin{aligned}
W_{U E}-W^{N} & =\sum_{k \in \mathcal{K}} S_{k}\left\{(1+\lambda)(p-e)\left(1-\beta_{k}\right) \int_{\underline{\theta}_{k}}^{\theta_{k}} \theta f_{k}(\theta) d \theta\right. \\
& \left.+\int_{\theta_{\ell^{\prime} E}}^{\theta_{k}}\left[(1+\lambda)\left(\hat{e}_{k} \theta-\hat{c}_{k}\right) f_{k}(\theta)+\left(\lambda-\alpha_{k}\right) \hat{p}_{k} F_{k}(\theta)\right]\right\} d \theta
\end{aligned}
$$

\subsection{Données et résultats numériques}

Les données sont issues du réseau d'information comptable agricole, qui est une collecte harmonisée à l'échelle européenne de données micro-économiques nationales. Outre les paramètres définis dans le corps du texte $\left(S_{k}\right.$ les surfaces en millions d'hectares, $\beta_{k}$ les contributions des États membres au budget européen, les paramètres $\hat{c}_{k}$ et $\mu_{k}$ définissant les coûts exprimé en $1000 \mathrm{ECU} / \mathrm{ha}$ ), nous indiquons dans le tableau 1 les parités de pouvoir d'achat (SPA), l'espérance et la variance nationales des rendements en blé (respectivement $E \theta_{k}$ et $V \theta_{k}$, les rendements étant exprimés en tonnes/ha), et les parts nationales de la demande européenne de blé tendre $\left(d_{k}\right)$.

Tableau 1. Données pour les simulations

\begin{tabular}{|l|l|l|l|l|l|l|l|l|}
\hline Pays & $\begin{array}{l}\text { SPA94 } \\
(\text { monnaie/ } \\
\text { ECU) }\end{array}$ & $\begin{array}{l}S_{k} \\
(\text { Mha })\end{array}$ & $\beta_{k}$ & $\begin{array}{l}E \theta_{k} \\
(\mathrm{t} / \mathrm{ha})\end{array}$ & $\begin{array}{l}V \theta_{k} \\
\left(\mathrm{t}^{2} / \mathrm{ha}^{2}\right)\end{array}$ & $\begin{array}{l}\hat{c}_{k} \\
(\mathrm{kECU} \\
\text { /ha })\end{array}$ & $\begin{array}{l}\mu_{k} \\
(\mathrm{kECU} \\
/ \mathrm{ha})\end{array}$ & $d_{k}$ \\
\hline$A L$ & 1.924 & 1.700 & 0.2635 & 6.2 & 9. & 0.75 & 0.035 & 0.2140 \\
$B E$ & 39.656 & 0.205 & 0.0410 & 7.0 & 8. & 14. & 0.6 & 0.0310 \\
$D K$ & 7.543 & 0.574 & 0.0215 & 6.1 & 7. & 2.5 & 0.1 & 0.0385 \\
$E S$ & 158.92 & 1.200 & 0.0770 & 3.20 & 5. & 28. & 1.25 & 0.0775 \\
$F R$ & 6.583 & 4.580 & 0.2040 & 6.3 & 10. & 2.3 & 0.1 & 0.2295 \\
$E L$ & .28803 & 0.78 & 0.0120 & 3.08 & 5. & 0.053 & 0.0025 & 0.0270 \\
$I R$ & 0.7936 & 0.074 & 0.0075 & 7.2 & 7. & 0.45 & 0.02 & 0.0105 \\
$I T$ & 1.915 & 0.7 & 0.1540 & 4.9 & 6.5 & 0.50 & 0.022 & 0.1575 \\
$L X$ & 39.656 & 0.007 & 0.0015 & 4.9 & 4. & 5.5 & 0.25 & 0.0005 \\
$N L$ & 2.158 & 0.123 & 0.0595 & 8.0 & 7. & 1.1 & 0.05 & 0.0390 \\
$P T$ & 196.9 & 0.22 & 0.0110 & 1.96 & 3. & 37. & 2. & 0.0170 \\
$U K$ & 0.7759 & 1.850 & 0.1475 & 7.0 & 9. & 0.35 & 0.014 & 0.1580 \\
\hline
\end{tabular}


Le tableau 2 indique d'une part les rendements seuils déterminant les producteurs en activité dans la situation de référence (en l'absence de politique de gel de terre). Il caractérise d'autre part les contrats de gel de terre en termes de rendement seuil, de part de surface gelée et de prime (la surface gelée étant le complément à 1 des surfaces en production). Le prix optimisant le bien-être social européen est également donné en première ligne (1000 ECU94/t).

Enfin, le tableau 3 donne d'une part les offres et demandes de bien calculées pour chacun des États membres, et d'autre part une évaluation du surplus affectant les consomateurs, les producteurs et les contribuables. Pour ces derniers, on distingue ce qui relève du budget national (les primes du gel subsidiaire) et ce qui relève du budget communautaire (primes du gel uniforme et restitutions aux exportations). La FBES indique la somme pondérée des surplus, en tenant compte de la préférence sociale accordée aux producteurs et du coût d'opportunité des fonds publics.

Tableau 2. Rendements seuils de production ( $t / h a$ ), surface en production (\%) et primes de gel (SPA94/ha)

\begin{tabular}{|c|c|c|c|c|c|c|c|}
\hline & Hors gel & \multicolumn{3}{|c|}{ gel subsidiaire $p=0.140$} & \multicolumn{3}{|c|}{ gel commun $\mathrm{p}=0.147$} \\
\hline Pays & rendement & rendement & surface & prime & rendement & surface & prime \\
\hline$A L$ & 2.957 & 3.518 & 91.823 & .040 & 4.077 & 81.933 & .134 \\
\hline$B E$ & 2.618 & 2.845 & 99.264 & .003 & 4.077 & 89.162 & .183 \\
\hline$D K$ & 2.424 & 2.614 & 98.824 & .001 & 4.077 & 79.345 & .212 \\
\hline$E S$ & 1.240 & 1.367 & 96.201 & .005 & 4.077 & 27.114 & .389 \\
\hline$F R$ & 2.592 & 2.994 & 95.669 & .025 & 4.077 & 78.931 & .186 \\
\hline$E L$ & 1.302 & 1.398 & 96.639 & .000 & 4.077 & 26.037 & .378 \\
\hline$I R$ & 4.544 & 4.910 & 93.907 & .000 & 4.544 & 100.000 & .000 \\
\hline$I T$ & 1.885 & 2.139 & 96.872 & .014 & 4.077 & 57.581 & .290 \\
\hline$L X$ & 0.965 & 1.040 & 99.995 & .001 & 4.077 & 60.694 & .433 \\
\hline$N L$ & 4.019 & 4.426 & 97.641 & .009 & 4.077 & 99.725 & .000 \\
\hline$P T$ & 1.344 & 1.440 & 93.363 & .000 & 4.077 & 15.926 & .368 \\
\hline$U K$ & 3.418 & 3.884 & 94.841 & .024 & 4.077 & 92.267 & .073 \\
\hline
\end{tabular}


Pierre-Alain Jayet, Gilles Rotillon 427

Tableau 3. Décomposition du bien-être social (BES, en millions d'ECU)

Tableau 3.1. Hors gel

\begin{tabular}{|l|r|r|r|r|r|r|r|r|}
\hline pays & demande & offre & surplusC & profit & budgetN & budgetUE & budgetT & BES \\
\hline$A L$ & 11.7700 & 10.8686 & .0000 & .7699 & .0000 & .2439 & .2439 & .5968 \\
$B E$ & 1.7050 & 1.4204 & .0000 & .1192 & .0000 & .0380 & .0380 & .0921 \\
$D K$ & 2.1175 & 3.5120 & .0000 & .2900 & .0000 & .0199 & .0199 & .3256 \\
$E S$ & 4.2625 & 4.1260 & .0000 & .3750 & .0000 & .0713 & .0713 & .3573 \\
$F R$ & 12.6225 & 29.0204 & .0000 & 2.3120 & .0000 & .1889 & .1889 & 2.5536 \\
$E L$ & 1.4850 & 2.6291 & .0000 & .2280 & .0000 & .0111 & .0111 & .2624 \\
$I R$ & .5775 & .5638 & .0000 & .0284 & .0000 & .0069 & .0069 & .0249 \\
$I T$ & 8.6625 & 3.4983 & .0000 & .3018 & .0000 & .1426 & .1426 & .1682 \\
$L X$ & .0275 & .0342 & .0000 & .0039 & .0000 & .0014 & .0014 & .0029 \\
$N L$ & 2.1450 & .9844 & .0000 & .0622 & .0000 & .0551 & .0551 & -.0014 \\
$P T$ & .9350 & .6240 & .0000 & .0459 & .0000 & .0102 & .0102 & .0417 \\
$U K$ & 8.6900 & 13.1488 & .0000 & .9006 & .0000 & .1366 & .1366 & .9064 \\
\hline$U E$ & 55.0000 & 70.4300 & .0000 & 5.4369 & & & .9258 & 5.3306 \\
\hline
\end{tabular}

Tableau 3.2. Gel subsidiaire

\begin{tabular}{|l|r|r|r|r|r|r|r|r|}
\hline pays & demande & offre & surplusC & profit & budgetN & budgetUE & budgetT & FBES \\
\hline$A L$ & 11.9995 & 10.4166 & .1159 & .6684 & .0055 & .1693 & .1748 & .6859 \\
$B E$ & 1.7382 & 1.4163 & .0168 & .1054 & 0.0000 & .0263 & .0263 & .1083 \\
$D K$ & 2.1588 & 3.4949 & .0208 & .2558 & 0.0000 & .0138 & .0138 & .3133 \\
$E S$ & 4.3456 & 4.0664 & .0420 & .3352 & .0002 & .0495 & .0497 & .3809 \\
$F R$ & 12.8686 & 28.4641 & .1243 & 2.0338 & .0050 & .1311 & .1360 & 2.4127 \\
$E L$ & 1.5140 & 2.5936 & .0146 & .2025 & .0000 & .0077 & .0077 & .2507 \\
$I R$ & .5888 & .5425 & .0057 & .0230 & .0000 & .0048 & .0048 & .0270 \\
$I T$ & 8.8314 & 3.4541 & .0853 & .2680 & .0003 & .0989 & .0993 & .2730 \\
$L X$ & .0280 & .0342 & .0003 & .0036 & 0.0000 & .0010 & .0010 & .0033 \\
$N L$ & 2.1868 & .9721 & .0211 & .0526 & 0.0000 & .0382 & .0383 & .0317 \\
$P T$ & .9532 & .6037 & .0092 & .0399 & .0000 & .0071 & .0071 & .0480 \\
$U K$ & 8.8595 & 12.7991 & .0856 & .7749 & .0022 & .0948 & .0970 & .8942 \\
\hline$U E$ & 56.0725 & 68.8576 & .5415 & 4.7633 & & & .6557 & 5.4290 \\
\hline
\end{tabular}


Tableau 3.3. Gel commun

\begin{tabular}{|l|r|r|r|r|r|r|r|r|}
\hline pays & demande & offre & surplusC & profit & budgetN & budgetUE & budgetT & FBES \\
\hline$A L$ & 11.8512 & 9.7770 & .0407 & .7531 & .0000 & .3273 & .3273 & .5004 \\
$B E$ & 1.7168 & 1.3422 & .0059 & .1159 & .0000 & .0509 & .0509 & .0759 \\
$D K$ & 2.1321 & 3.1090 & .0073 & .2886 & .0000 & .0267 & .0267 & .3217 \\
$E S$ & 4.2919 & 1.9718 & .0148 & .5568 & .0000 & .0956 & .0956 & .5595 \\
$F R$ & 12.7096 & 25.7301 & .0437 & 2.2966 & .0000 & .2534 & .2534 & 2.4881 \\
$E L$ & 1.4952 & 1.2089 & .0051 & .3475 & .0000 & .0149 & .0149 & .4078 \\
$I R$ & .5815 & .5638 & .0020 & .0265 & .0000 & .0093 & .0093 & .0212 \\
$I T$ & 8.7223 & 2.5853 & .0300 & .3300 & .0000 & .1913 & .1913 & .1644 \\
$L X$ & .0277 & .0255 & .0001 & .0042 & .0000 & .0019 & .0019 & .0026 \\
$N L$ & 2.1598 & .9831 & .0074 & .0588 & .0000 & .0739 & .0739 & -.0244 \\
$P T$ & .9415 & .2038 & .0032 & .0893 & .0000 & .0137 & .0137 & .0929 \\
$U K$ & 8.7500 & 12.6094 & .0301 & .8608 & .0000 & .1832 & .1832 & .8228 \\
\hline$U E$ & 55.3795 & 60.1099 & .1904 & 5.7280 & & & 1.2421 & 5.4329 \\
\hline
\end{tabular}

\section{Références}

Baron D.P. et R.B. Myerson, (1982), "Regulating a monopolist with unknown costs", Econometrica, 50, pp. 911-930.

Bourgeon J.-M., P.-A. Jayet et P. Picard, (1995), "An incentive approach to land set-aside programs", European Economic Review, 39, pp. 14871509.

Jayet P.-A., (2001), Évaluation de politiques de retrait des terres pour la régulation d'un marché agricole, Annales d'économie et de statistique, 61, pp. 71-102.

Gisser M., (1993), "Price support, acreage controls and efficient redistribution", Journal of Political Economy, 101, pp. 584-611.

Union Européenne, (1998), Pac 2000-situation et perspectives-vin, Technical report, Office des publications officielles des communautés européennes. 